\title{
Tackling the hurdles of electrically pumped colloidal quantum dot lasers
}

\author{
Yue Wang ${ }^{1,2}$ and Handong Sun ${ }^{1,2^{*}}$
}

Lasers are devices that produce light by stimulated emission (SE) which amplifies light coherently. They are distinguished from other light sources by their beams with good coherence, directionality, high brightness and spectral purity. As such, lasers have widespread applications, including scientific research, industry, medicine and military defense, and actually become ubiquitous in our daily life. Despite of the diversity in laser classification, compact lasers are dominantly made from semiconductors. Especially, electrically driven semiconductor lasers are indispensable components for optical communication. At present, electrically driven semiconductor lasers, or laser diodes, are fabricated by the high-vacuum and high-temperature epitaxy methods, which are not only costly but also difficult to integrate with other optoelectronic devices [1]. As a complement to the epitaxial semiconductor lasers, the cost-effective solution-processed laser diodes have been pursued for the past decades yet demonstrated to be extremely challenging.

Colloidal quantum dots (CQDs), or nanocrystals, are tiny semiconductor particles whose size lies in the range of nanometers [2]. By virtue of the quantum confinement effect, CQDs exhibit size-tunable emission colors, delta function-like electronic density of states and large optical oscillator strength, endowing them with superior optical properties. Moreover, the synthesis of CQDs relies on the simple wet-chemistry process and the resultant CQD suspension enables the fabrication of devices by the facile solution-based methods, such as inkjet printing and spincoating. Ever since the first realization of SE in 2000, CQDs have been envisaged as the attractive candidates towards the solution-processed laser diodes [3]. However, until now, all of the SE and lasing demonstrations from CQDs are exclusively based on optical pumping, while electrically driven lasing as required in most applications remains to be unaccomplished $[3,4]$.

The challenges to achieve electrically pumped CQD laser are multifold and complex, but have been much better understood now thanks to the previous comprehensive optical studies. First, in CQDs, the band-edge level has double degeneracy, so that the population inversion which is required for lasing can only be achieved when the average number of excitons per CQD, $\langle N\rangle$, is larger than one [1]. In this situation, the nonradiative Auger recombination (AR) would be activated, which is exceedingly fast due to the enhanced carrier-carrier interaction in CQDs. Such AR has proved to be the main gain depletion channel for the optically pumped CQD lasing, and it will plague the electrically driven lasing more seriously as carriers are injected into CQD one-byone $[3,4]$. Consequently, suppressing AR is a key step toward electrically pumped CQD lasers. Second, different from epitaxially grown heterostructures which employ a p-n junction to inject current for excess carrier generation, CQDs rely on an electron transport layer and a hole transport layer for carrier injection. Limited by material selection, excess carrier generation through current injection into CQDs is quite inefficient. In order to achieve population inversion through electrical injection in CQDs, it is necessary to inject a high current (tens of $\mathrm{A} \mathrm{cm}^{-2}$ ) through the CQD layer, which would bring about serious issues. On the one hand, organic ligands are overwhelmingly employed to passivate the CQD surface and maintain the favorable colloidal stability. However, these surface ligands, typically possessing poor conductivity, will block the carrier injection and transportation in CQDs. On the other hand, the device can be easily damaged by Joule heating induced temperature increase under such high excitation densities during electrical injection, a notorious issue in laser diodes [1]. Thus effec-

\footnotetext{
${ }^{1}$ Division of Physics and Applied Physics, School of Physical and Mathematical Sciences, Nanyang Technological University, Singapore 637371, Singapore

${ }^{2}$ Centre for Disruptive Photonic Technologies (CDPT), Nanyang Technological University, Singapore 637371, Singapore

*Email: hdsun@ntu.edu.sg
} 


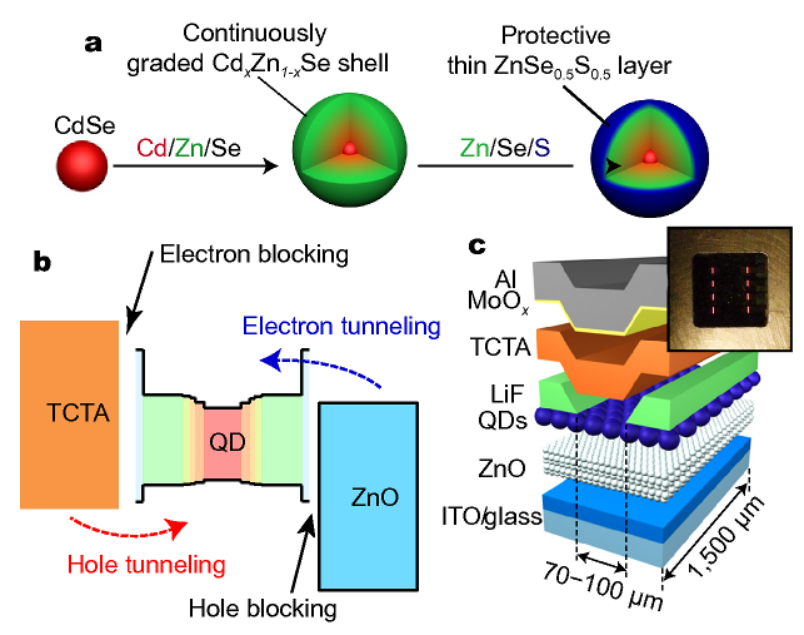

Figure 1 (a) Schematic illustration of the fabrication flow and structure of the compositionally graded CdSe/ $\mathrm{Cd}_{x} \mathrm{Zn}_{1-x} \mathrm{Se}_{\mathrm{ZnSe}} \mathrm{Zn}_{0.5} \mathrm{~S}_{0.5}$ quantum dots adopted for the electrical pumped optical gain. (b) A simplified band diagram of the device illustrating that electrons are injected from the $\mathrm{ZnO}$ layer, while holes are injected from the layer of tris(4-carbazoyl-9ylphenyl)mine (TCTA). (c) The p-i-n architecture adopted in the work, a specially shaped hole-injecting layer with a small-area contact (70-100 $\mu \mathrm{m}$ wide) to the CQD layer, enabling the high current density up to $18 \mathrm{~A} \mathrm{~cm}^{-2}$. The inset shows the photograph of a real-life 8-pixel device under applied bias. Reprinted with permission from Ref. [5], Copyright 2017, Nature Publishing Group.

tive thermal management plays an important role in electrically pumped CQD lasers.

Recently, Lim and his colleagues from the Los Alamos National Laboratory in USA reported significant progress in tackling the above long-standing challenges [5]. In terms of AR suppression, they engineered the novel $\mathrm{CdSe} / \mathrm{Cd}_{x} \mathrm{Zn}_{1-x} \mathrm{Se}_{\mathrm{ZnSe}} \mathrm{Zn}_{0.5}$ CQDs with alloyed core/shell interface (Fig. 1a), based on which they obtained long optical gain lifetime of nanosecond level and low SE threshold upon photo-excitation. Such interface grading strategy has been theoretically and experimentally demonstrated to be effective in suppressing AR for other CQD systems [6-8]. Regarding the electrical pumping, they exploited an architecture using optimized hybrid charge transport layers (Fig. 1b). Most importantly, they applied a particularly shaped insulating spacer between the CQD layer and charge-injection layers to form the "current-focusing" structure and the active CQD layer is made as thin as 1-2 monolayers (Fig. 1c). The special device configuration has twofold advantages and may be the key for the success. First, such device enables the high current injection $\left(18 \mathrm{~A} \mathrm{~cm}^{-2}\right.$, equivalent to $\left.\langle N\rangle=3.2\right)$ required for population inversion. Second, the designed structure allows efficient heat dissipation from the active area, thereby overcoming heating effect. By the above efforts, they, for the first time, achieved the electrically pumped optical gain from CQDs.

This work certainly removed the hurdles of electrically pumped optical gain in CQDs and opens the door to the realization of the solution-processed CQD lasers. Although the demonstrated strategy of reducing AR rate by gradient interface and the efficient current injection was manifested in II-VI group metal-chalcogenide semiconductor quantum dots, hopefully it can be applied to other material systems such as III-V group semiconductors and the emerging halide perovskites. The next effort would be to engineer proper optical cavity to provide the optical feedback and eventually to realize electrically injected lasers from CQDs.

Received 12 December 2017; accepted 14 December 2017; published online 22 December 2017

1 Nathan MI, Dumke WP, Burns G, et al. Stimulated emission of radiation from GaAs p-n junctions. Appl Phys Lett, 1962, 1: 62-64

2 Liu M, Wang K, Wang L, et al. Probing intermediates of the induction period prior to nucleation and growth of semiconductor quantum dots. Nat Commun, 2017, 8: 15467

3 Klimov VI, Mikhailovsky AA, Xu S, et al. Optical gain and stimulated emission in nanocrystal quantum dots. Science, 2000, 290: 314-317

4 Wang Y, Ta VD, Gao Y, et al. Stimulated emission and lasing from $\mathrm{CdSe} / \mathrm{CdS} / \mathrm{ZnS}$ core-multi-shell quantum dots by simultaneous three-photon absorption. Adv Mater, 2014, 26: 2954-2961

5 Lim J, Park YS, Klimov VI. Optical gain in colloidal quantum dots achieved with direct-current electrical pumping. Nat Mater, 2017, 4: $42-49$

6 Wang Y, Leck KS, Ta VD, et al. Blue liquid lasers from solution of $\mathrm{CdZnS} / \mathrm{ZnS}$ ternary alloy quantum dots with quasi-continuous pumping. Adv Mater, 2015, 27: 169-175

7 Wang Y, Fong KE, Yang S, et al. Unraveling the ultralow threshold stimulated emission from CdZnS/ZnS quantum dot and enabling high-Q microlasers. Laser Photonics Rev, 2015, 9: 507-516

8 Nasilowski M, Spinicelli P, Patriarche G, et al. Gradient CdSe/CdS quantum dots with room temperature biexciton unity quantum yield. Nano Lett, 2015, 15: 3953-3958 